\title{
Horizontal well workover technology present situation and development trend
}

\author{
Guohui Zhang ${ }^{1, *}$, Pengwang ${ }^{1}$, Rongchen ${ }^{1}$, Baoping Duan ${ }^{1}$, Guowen Zhang ${ }^{1}$, and Jiewen Sun ${ }^{1}$ \\ ${ }^{1}$ PetroChina Research Institute of Petroleum Exploration \& Development, Beijing 100083, China
}

\begin{abstract}
In the downhole operation of horizontal Wells, the pipe string is subjected to the combined action of many kinds of loads. The pipe string may bend sinusoidal or spiral under the action of axial compressive stress, which will also increase the friction between the pipe string and the wellbore wall. Because of the existence of friction, to the milling, salvage, unstuck, sand and other construction to bring adverse effects. This paper describes the characteristics of horizontal well in fishing, sand washing, unlocking and casing milling. The paper introduces the development of horizontal well application, such as drilling technology matching for complex structure wells, high-level completion of lateral Wells, radial lateral wells in low permeability reservoirs and supporting technology for completion and production of horizontal wells.
\end{abstract}

\section{Introduction}

In the downhole operation of horizontal well, the pipe string is subjected to the combined action of many kinds of loads, including the internal pressure, external pressure, fluid viscous force inside and outside the pipe string, the supporting reaction force of the casing wall, and the friction force of the casing ${ }^{[1-2]}$. The string can bend sinusoidally or helically under axial compressive stress, which also increases the friction between the string and the wellbore. When the friction is high enough, selflocking can occur. Due to the large friction force, to the milling, salvage, unjam, sand and other construction to bring adverse effects. The strength of pipe string decreases with the increase of wellbore temperature, and the instability phenomena such as bending, deformation and buckling will occur when the force of pipe string exceeds the strength range. Horizontal well workover started late in China, and the technology lagged behind ${ }^{[3-6]}$. The stress analysis and application research of downhole pipe string need to be improved urgently. In the development of oil and gas fields, the difficulty and number of horizontal well workover are increasing continuously. Different from the traditional vertical well, horizontal well has a special well trajectory, and the downhole pipe string is subjected to a complex force, and the wear, resistance and deformation caused by it are more serious. Therefore, the workover technology of horizontal well will face great challenges. In the workover operation, the understanding of the force, especially the resistance, on the string in the casing is not perfect. Horizontal Wells in the process of operation, the downhole string subjected to complicated stress state, only through the mouth of the well load changes, is difficult to judge the downhole string actual bearing, with experience of operation, simple is difficult to guarantee the construction safety and construction quality, need to establish a more comprehensive string stress analysis model of horizontal well and develop the corresponding mechanical analysis software ${ }^{[7-11]}$.

\section{Horizontal well workover technology}

In horizontal well workover operation engineering, stress changes of pipe string in various links such as drilling, completion and workover should be taken into account, and research on horizontal well workover and each link technology should be strengthened to provide a reliable guarantee for the application of horizontal well workover technology in oil field ${ }^{[12]}$.

\subsection{Horizontal well fishing technology}

Compared with vertical Wells, horizontal Wells have more complex forces on the operating string due to the existence of curved section and horizontal section. The lifting force of the wellhead of a horizontal well cannot be effectively transferred to the horizontal section under the action of the deflection section, and the fishing technology such as vigorous lifting and movable unlocking cannot be implemented in a horizontal well, because it will cause wear and tear on the casing, and the risk of the pipe string itself breaking down the well is also high.

It is not practical to carry out conventional fishing techniques such as casing milling and reversing in horizontal wells. Because of the existence of deflection section, the wellhead torque is difficult to be effectively transferred to the horizontal section. Horizontal perforation section of horizontal wells is relatively long, and the length of sand filter pipe reaches tens or even hundreds of meters. Because the sand filter pipe is equipped with a centralizer, the difficulty of salvage is

\footnotetext{
* Corresponding author: zhangguohui@ petrochina.com.cn 
increased relative to the enlargement of the pipe body.

In order to solve the above problems, a redesign of the fishing string structure was adopted. By adding some necessary tools, direct tension in nearby, implementation solution of horizontal well, thus improve the salvage possibilities. At present, it has a relatively mature technology of salvaging small falling objects and pipes, and the salvaging objects mainly include packer, wire winding pipe, sand filter pipe and small falling objects. Every fishing operation in horizontal well is not isolated, should consider the actual situation, such as borehole conditions, the causes for the stuck drill, drill and ultimate strength equipment hoisting capacity, etc.

Fishing string structure:drill pipe + slope drill tool + mechanical (hydraulic) type fishing reverse tool. Overhead fishing string structure:

(1) Drill pipe + ramp drill tool + mechanical reverse switch + fishing tool;

(2) drill pipe (tubing)+ slope drill tool + hydraulic type reverse switch + fishing tools;

(3) Back-up drill pipe + back-up slope drill tool + hydraulic safety joint + fishing back-up tool.

\subsection{Horizontal well sand washing technology}

In the sand control operation of horizontal well, the sand produced by the formation and the filling sand in the horizontal wellbore section have its characteristics:

(1) In the process of sand washing, particles are easy to deposit, and the sedimentation rate is greatly affected by the particle size and the density difference between particles and the sand-carrying fluid.

(2) When the horizontal well is sanding, it is easy to form dunes at the bottom of the horizontal section. In severe cases," sand bed" in the long well section is formed, and even sand stick is caused.

(3) During the sand recoil construction, some sand grains were deposited at the bottom of the horizontal section of the tubing, which could not be carried out of the wellbore. In the process of pulling up the string, the deposited sand will flow back into the wellbore, resulting in incomplete sand washing and unable to meet the construction requirements.

According to the characteristics of sand washing in horizontal Wells, some researches have improved the continuous sand washing tool and designed the spiral groove sand washing device, which can form the water vortex to wash up the sand attached to the horizontal section, and realize the continuous, fast and efficient sand washing. The continuous sand washing tool is composed of a flushing pen tip, a tool body, a spiral flume and an upper joint. The direction of fluid flow changes from straight line flow through the spiral flume of the tool to spiral eddy current, and the settling sand at the junction of the vertical and horizontal well sections in the wellbore is flushed up and returned to the surface with the sand washing fluid to meet the purpose of sand washing construction. During cyclic sand-washing, the tool can wash the settling sand at the junction of the vertical and horizontal sections through the swirling circulation of the sand-washing fluid, and the sand-washing fluid will carry the sand particles out of the wellbore and back to the surface in a turbulent manner.

Horizontal well sand flushing string structure: tubing + process pipe tubing + spiral centralizer + spiral groove sand flusher.

\subsection{Horizontal casing (grinding) milling technology}

(1) The wellhead torque loss caused by the friction of the horizontal well string during casing milling.

(2) The conventional casing milling pipe string produces large friction resistance, the equipment transmission system is easy to be damaged, and the workover fluid has low viscosity. In the casing milling construction, the cuttings, iron chips, ash chips and other sundries can not be well blown out of the wellbore, resulting in deposits in the horizontal section, which is easy to cause sticking accidents.

(3) In horizontal well construction, due to the influence of well trajectory, the load applied by casing milling can not be fully transferred to the front-end tool, leading to the reduction of casing milling efficiency.

(4) The following problems may occur during conventional milling:

A. Due to wear of horizontal well casing, it is difficult to mill large metal falling objects, with long cycle and high risk.

B. Fragments generated by milling will lead to stuck pipe accidents and increase the risk of subsequent construction. Considering the influence of borehole curvature, the length of casing milling pipe is generally not more than $10 \mathrm{~m}$, and the casing milling pipe is machined from $116 \mathrm{~mm}$ or $127 \mathrm{~mm}$ pipe. Ramp drilling tools are used in horizontal Wells, workover fluid is tested according to the requirements of construction standards to ensure that all indicators of workover fluid meet the requirements of construction, and anti-deviant wear drill pipe collar is used every $30 \mathrm{~m}$ in deviation section of the well. The centralizer tools include ball centralizer, spiral centralizer, etc. Improved casing milling pipe string combination:(top down) kelly+drill pipe+slope drill pipe+anti-deviation grinder+fluid rheometer +casing milling tool. The casing milling string with this structure combination is more suitable for horizontal wells and highly deviated wells. The oblique groove on the rheological vectorator changes the flow direction of workover fluid and forms swirling flow state, which improves the carrying capacity of workover fluid, reduces the settling speed of the milling debris, and makes it easier for the milling debris to break out of the wellbore and return to the surface.

Because the milling debris is not easy to discharge, when reaching a certain footage (milling sand filter pipe 4 $\sim 5 \mathrm{~m}$ ), there will be a phenomenon of holding back drilling, drilling and milling should be stopped, the pipe string should be removed, the debris in the well should be cleaned up through reverse circulation flushing, and then milling or fishing. 


\subsection{Horizontal well sticking technology}

Due to the well trajectory of horizontal wells, the surface load cannot be effectively transferred to the horizontal section of the well, and the implementation effect of horizontal well fishing is poor. The sand filter pipe of horizontal well is relatively long, and the outer diameter of the centralizer of the sand filter pipe is large. After sticking, it is difficult to implement such technical measures as casing milling and bucking, and the difficulty of fishing is further increased.

Hydraulic force lift technology is a new technology to solve the problem of axial force transfer and reduce the risk of string fracture. The hydraulic booster is composed of three parts, namely: (bottom to top) horizontal pressure ball seat + force part + casing anchor part. ZLQ-114 hydraulic augmented fishing device uses the hydraulic pressure in the drilling tool to push the five-stage piston up, which can produce a large lift force. The main engine with the ZLSLM-114 and ZLSLM-150 hydraulic anchors can be used for the lifting of $\Phi 139.7 \mathrm{~mm}$ casing and $\Phi 177.8 \mathrm{~mm}$ casing. Horizontal pressure ball seat can ensure that the tool is pressed in a horizontal state, while the pressure can be relieved according to the construction needs. The augmentation part is the part that produces the tension directly. The role of the casing anchoring part is to anchor the downhole fishing booster to the casing. Auxiliary tools include constant pressure discharge circulation valve, guard type retractable spear and other tools.

By means of hydraulic transmission, the hydraulic augmentator can compensate for the lack of tensile strength of drilling tools and lifting capacity of surface equipment, and also solve the transfer problem of unstuck force in large deviation section and horizontal section, so as to achieve the purpose of unstuck. The technology adopted is: "washing", "dredging", "loosing" and "pulling". The sand filter pipe can reach $200 \mathrm{~m}$ at one time, and the process efficiency is more than $90 \%$. It is the leading technology for the sticking of horizontal Wells in oil fields at present. The reverse fishing technology is put forward after the pipe string above the stuck point is reversed one by one. It is a kind of fishing technology to gradually solve the problem of salvages and fallen objects. The new type of hydraulic reverse fishing device with large torque increases the torque from the original 5 $6 \mathrm{kN} . \mathrm{m}$ to $20 \mathrm{kN} . \mathrm{m}$. It is safe and reliable with high efficiency by using mechanical buckling device and straight buckling tool to realize buckling of falling pipe string. For those Wells with serious sand production, which can't be salvaging with downhole augmenting force, the technology of casing milling and inverted fishing can be adopted. At present, the reverse trip technology used in horizontal Wells is the same as that used in vertical wells. The wellhead is driven by rotary table and the combination of reverse trip drill pipe+chamfered drill pipe+centralizer+reverse trip fishing tool is used to reverse trip. The technology of vertical Wells is directly used in horizontal Wells, which has some shortcomings such as inaccurate reverse trip position and excessively large torque of drilling tool.
The horizontal well fishing string consists of a combination of the following: (top-down) wellhead highpressure self-sealing well capper, downhole fishing force booster, and lift-down retrievable spear.

\subsection{Casing protection technology for horizontal Wells}

(1) add graphite powder, paraffin, white oil and other lubricants into the workover fluid, form lubricating layer between the drill tool and the well wall, effectively reduce the friction coefficient and form a low-friction workover fluid.

(2) Optimize the BHA by using sloped BHA for high deviations and horizontal sections; various types of rolling centralizers are used to change sliding friction into rolling friction to reduce damage to casing caused by rolling, pulling and unjamming friction.

(3) the set of milling tool body hard alloy is not exposed, the use of formal and efficient set of milling tools speed up the construction speed, reduce casing damage; Reverse circulation is preferred to improve carriability and minimize debris generation out of the wellbore.

(4) The application of hydraulic tools for construction can effectively protect the casing above the tool from damage.

\section{Development direction of horizontal well application}

\subsection{Complicated structure well drilling technology}

Complex structure well is a series of well types with horizontal or branching characteristics, including horizontal well, branching well, extended reach well, fishbone well and connected well, etc. The drilling technology of complex structure well in foreign countries is becoming more and more perfect and mature, and the supporting technology of complex structure well drilling is gradually formed, which takes optimization design, well trajectory control, measurement while drilling, new downhole tools, geosteering drilling, rotary steering drilling system and so on as the core, and the success rate of horizontal well drilling reaches $90 \% \sim 95 \%$. The drilling footage of horizontal wells in the United States increased from 2.3774 million meters in 2000 to 22.9514 million meters in 2008, an increase of 8.65 times. At present, the drilling footage accounts for $33.06 \%$ of the total footage, and the horizontal displacement of extended-reach wells has reached $10,805 \mathrm{~m}$. At present, horizontal well, lateral well, extended reach well and other complex structure wells have been used in more than 70 countries and more than 2300 oil fields in the world. Compared with foreign countries, China started late in the aspect of extended reach wells, and there is still a big gap between China and the world's advanced level. Domestic shale gas drilling is just beginning. With the rapid development of rotary steerable drilling technology in China, the drilling technology with complex structure is 
becoming mature again.

\subsection{High-level completion technology for lateral Wells}

A lateral is a primary well and two or more lateral wellbores, each of which can be drilled individually or in combination.

\subsection{1 lateral horizontal Wells}

The total horizontal section length of multi-branch horizontal wells abroad has reached $12300 \mathrm{~m}$. In China, multi-lateral wells are mainly distributed in Bohai Bend Basin, Shengli Oilfield, Liaohe Oilfield and Xinjiang. In recent years, China has made great progress in multilateral well completion, and developed some completion systems with independent intellectual property rights. However, compared with foreign advanced well completion technologies, most of them are designed for a certain oil and gas field and lack general applicability, so it is difficult to be widely promoted. In terms of technology, there is still a large gap between China and foreign countries in solving the isolation problem of multi-branch wellbore, which is mainly reflected in the lack of intelligent degree and poor controllability of downhole tools for well completion. Branch well drilling technology has been successfully applied in Shengli Oilfield. A twobranch horizontal well was successfully put into operation in the first block of the pile. This block belongs to a high water and thick bottom water reservoir. The two laterals were fully cemented with a post-perforated, in-pipe sand control completion method and an optional production process string. After put into production, compared with the surrounding horizontal Wells, the initial oil production reached $10 \mathrm{t}$ per day, and the initial water cut was 9 percentage points lower. In the future production process, the water cut rose slowly,2.5 times as much as that of the horizontal wells with the same oil production per day. Especially, the advantages of pressing bottom water coning were more obvious, and the advantages of increasing oil in the later stage were obvious. Fluid volume reached up to 1.8 times that of horizontal wells.

\subsubsection{An open hole lateral horizontal well in the shape of a fishbone}

Since 2006, Shengli Oilfield has been actively exploring the fishbone open hole lateral horizontal well technology. Due to the relatively simple operation of this technology, a well has been drilled in an offshore field. Screen completion method was used to complete the main wellbore of the fishbone open hole lateral horizontal well, which greatly increased the drainage area. The designed scheme is a four-branch horizontal well with a length of $398 \mathrm{~m}$ in the main horizontal section. The target point and the main wellbore are set within about 6 meters from the top of the reservoir, which is the middle and lower part of the reservoir. The lengths of the horizontal sections of the four branches are $149 \mathrm{~m}, 154 \mathrm{~m}, 149 \mathrm{~m}$ and $120 \mathrm{~m}$ respectively, and the included angles with the main branch are all $20^{\circ}$. The accumulated horizontal section length of Chengbei 26B-Ping1 fishbone open hole branch horizontal well is $919 \mathrm{~m}$, among which the main hole is $403 \mathrm{~m}$ long. The first lateral bore is $151 \mathrm{~m}$ long and has a maximum Angle of $13.55^{\circ}$. The second lateral hole is $136 \mathrm{~m}$ with a maximum Angle of $17.35^{\circ}$. The third lateral hole is $145 \mathrm{~m}$ with a maximum Angle of $27.54^{\circ}$. The fourth lateral hole is $84 \mathrm{~m}$ with a maximum Angle of $23.85^{\circ}$.After the completion of drilling, the calculated geological reserves are $60 \times 10^{4} \mathrm{t}$. The $5 \mathrm{~mm}$ nozzle is used for trial production, and the daily oil production is 94.8 tons.

\subsection{Radial lateral well technology for low permeability reservoirs}

Low permeability thin interbedded reservoirs have many oil layers, small single layer thickness and interlayer interference. Due to poor reservoir physical properties, serious heterogeneity, low natural productivity. The advantage of using highly deviated horizontal Wells is that the apparent thickness of thin reservoir can be greatly increased, thus the oil supply volume can be expanded, and the productivity of single well can be improved. For example, the apparent thickness of oil reservoir in 70Ping1 well is $62.5 \mathrm{~m}$, and the reservoir thickness is increased by nearly 5 times. The initial daily oil production is 22 tons, and the stable production is 12 tons, which is about $6 \sim 7$ times of that in vertical Wells. It has accumulated 4784 tons of oil and has obvious advantages.

\subsection{Supporting technology of horizontal well completion and oil production process}

In order to meet the development needs of horizontal well technology, it is necessary to further develop the supporting technology of horizontal well completion and oil production.

(1) gravel packing sand control technology in evacuated sandstone reservoir

Most of the horizontal wells in sand producing reservoirs abroad adopt gravel packing sand control technology, which has the advantages of good sand control effect and long working life. At present, this sand control method is not mature in horizontal wells, and the longest sand control method can only reach $100 \mathrm{~m}$, which is difficult to meet the field needs. Therefore, it is necessary to continue to study the key technologies and equipment of the gravel packing technology in the casing of horizontal Wells.

(2) Unconventional horizontal well fracturing technology

The multistage fracturing and completion technology of horizontal wells for unconventional oil and gas in foreign countries mainly forms the multistage fracturing and completion technology of horizontal wells with open hole packer and the multistage fracturing and perforation combined technology of pumping bridge plug. At present, the foreign subsection is up to 90 grades, the longest horizontal section is $4900 \mathrm{~m}$, the maximum sand addition in a single section is $450 \mathrm{~m} 3$, and the maximum liquid volume in a single section is $2550 \mathrm{~m}^{3}$. The two laterals were 
fully cemented with a post-perforated, in-pipe sand control completion method and an optional production process string. After put into production, compared with the surrounding horizontal wells, the initial oil production reached 10tons per day, and the initial water cut was 9 percentage points lower. In the future production process, the water cut rose slowly, 2.5 times as much as that of the horizontal Wells with the same oil production per day. Especially, the advantages of pressing bottom water coning were more obvious, and the advantages of increasing oil in the later stage were obvious. Fluid volume reached up to 1.8 times that of horizontal wells.

\section{4 conclusion}

At present, horizontal wells in domestic normal production account for about $10 \%$. Because the wellhead torque can not be effectively transferred to the horizontal section under the action of deflection section, vertical wells are used. Fishing techniques, such as back-up, casing milling,and milling, are relatively poor in horizontal Wells.

1. Downhole operation and construction problems of horizontal wells (especially milling and fishing, unblocking, sand control, fracturing and water plugging) are becoming more and more prominent. Under the dual action of large "pendulum force" and large friction area, the string is more likely to get stuck when it is close to the lower side of the well wall. In the horizontal section, sand beds tend to form, which increases the friction area of the string and thus the torque, resulting in complex well conditions.

2. As the well inclination increases, the degree to which the string axis deviates from the wellbore curve will increase, resulting in contact with the low side of the wellbore, which can easily cause differential stuck pipe. When sanding, plugging, and milling operations are performed in horizontal wells, the flow velocity of the wash fluid in the annulus varies greatly, resulting in a low flow velocity at the lower edge of the wellbore and a reduced sand-carrying capacity. When the pipe string is placed in the casing and does not move, it is easy to cause sticking accidents.

3. The lifting force on the wellhead of the horizontal well cannot be effectively transferred to the horizontal section underground. At this time, because of the effect of the deviating section of the horizontal well, the axial load will lose 1/3 2/3 due to the influence of the frictional force between the deviating section and the horizontal section. Under the influence of the friction force of the deflection section and the horizontal section, the value of torsion loss is related to the lifting force, and the loss is quite large. In the process of downhole operation, large load activities, such as unlocking and casing milling, which need to transfer large torsional data, will cause relatively large damage to casing.

\section{References}

1. Lubinski A. A Study of the Buckling of Rotary Drilling Strings[J]. API Drill and ProdPrac. 1950,
09:178-214

2. Lubinski A. and Woods H. B. Factors Affecting the Angle of Inclination and Dog-Legging in Rotary Bore Holes[J]. API Drill. and Prod. Prac. 1953, 08:57-60

3. Woods H. B. and Lubinski A. Practical Charts for Solving Problems on HoleDeviationll[J]. API Drill. and Prod. Prac. 1954, 07:8 5-88

4. Lubinski, A. and Blenkarn, K. A. Buckling of Tubing in Pumping Wells, Its Effects and Means for Controllinglt. Trans[J], AIME 1957, 12:21-24

5. Lubinski, A. and Althouse W. S. and Logan J. L. Helical Buckling of Tubing sealed inPackers[J]. JPT JUNE, 1962, 655-670

6. Hammerlind ID. J. Movement, Forces and Stresses Associated with CombinbationTubing Strings Sealed in Packers[J]. JPT, FEB. 1977, 12:195-203

7. Mitchell R. F. Comprehensive analysis for buckling with friction. SPE Drilling\&Completion, 1997, 12(1): 63-69

8. Yu-Che Chen and Yu-hsu Lin and Cheatham, J. B. Tubing and Casing Buckling inHorizontal Wells[J]. Pet, Tech. 1990, 140-141

9. Suman J. and Klementich E. F; etc. Measurement of Casing Buckling in ProductionIntervals[C]. SPE2647, 1993

10. Yu-che Chen, Sarmad Adnan Buckling of Pipe and Tubing Constrained Inside Inclined Wells presented at the 25 Annual Offshore Technology Conference inHouston. 1993

11. Miska, S and Cunha, J. C. An Analysis of Helical Buckling of Tubulars Subjected to Axial and Torsional Loading in Inclined Wellbores[C]SPE, 1995

12. Hill, T. H. Chandler, R. B. Field Curves for Critical Buckling Loads in Curving Wellbores[J]. SPE, 1998, 259-265 